\title{
Fermilab SpinQuest/E1039 experiment with Transversely Polarized Target
}

LANL : M. Yurov

E1039 SpinQuest collaboration

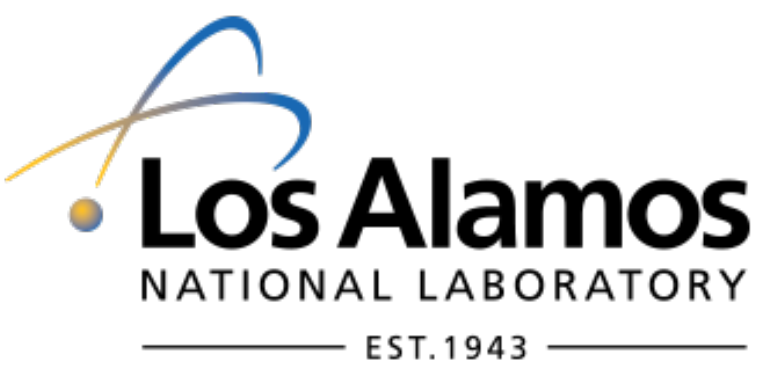

This manuscript has been authored by Fermi Research Alliance, LLC under Contract No. DEAC02-07CH11359 with the U.S. Department of Energy, Office of Science, Office of High Energy Physics. 52nd Annual Fermilab Users Meeting 06/13/19, Fermilab, Batavia, IL 


\section{QUEST for PROTON SPIN ORIGIN}

1987 EMC polarized DIS measurements:

contributions of spins of quarks and anti-quarks to the proton spin are small

broad world-wide program to resolve the problem of the nucleon spin composition

In spite of significant experimental and theoretical advances basic problem remains after 30 years of research

components contributing to proton spin

\section{$\Delta \Sigma \Delta G \quad L_{q} \quad L_{g}$}
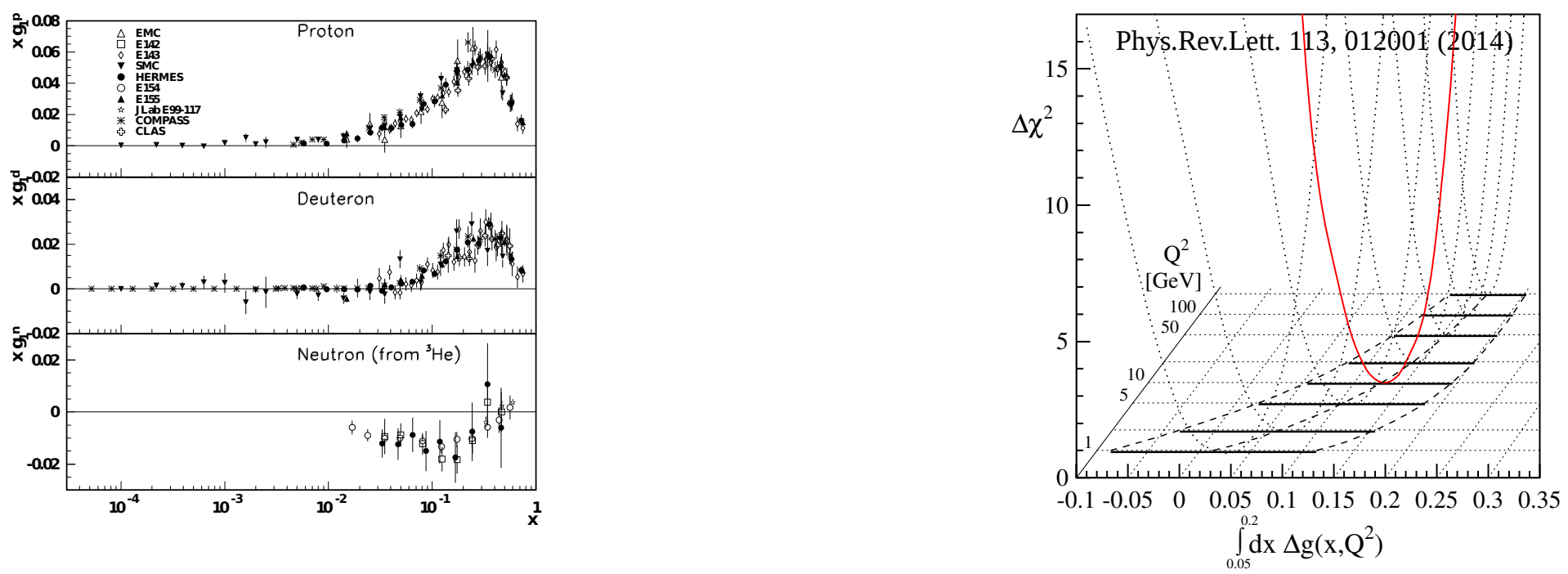


\section{WHY ARE WE DOING E1039?}

$$
\frac{1}{2}=\frac{1}{2} \Delta \Sigma+L_{q}+L_{\bar{q}}+\Delta G+L_{g}
$$

importance of sea quarks Lattice QCD: K.-F. Liu et al arXiv:1203.6388

- account for spin of quarks, anti-quarks and gluons - still missing about half the picture

- sea quark OAM could be a major part of missing spin

contribution of OAM of sea quarks remains poorly constrained

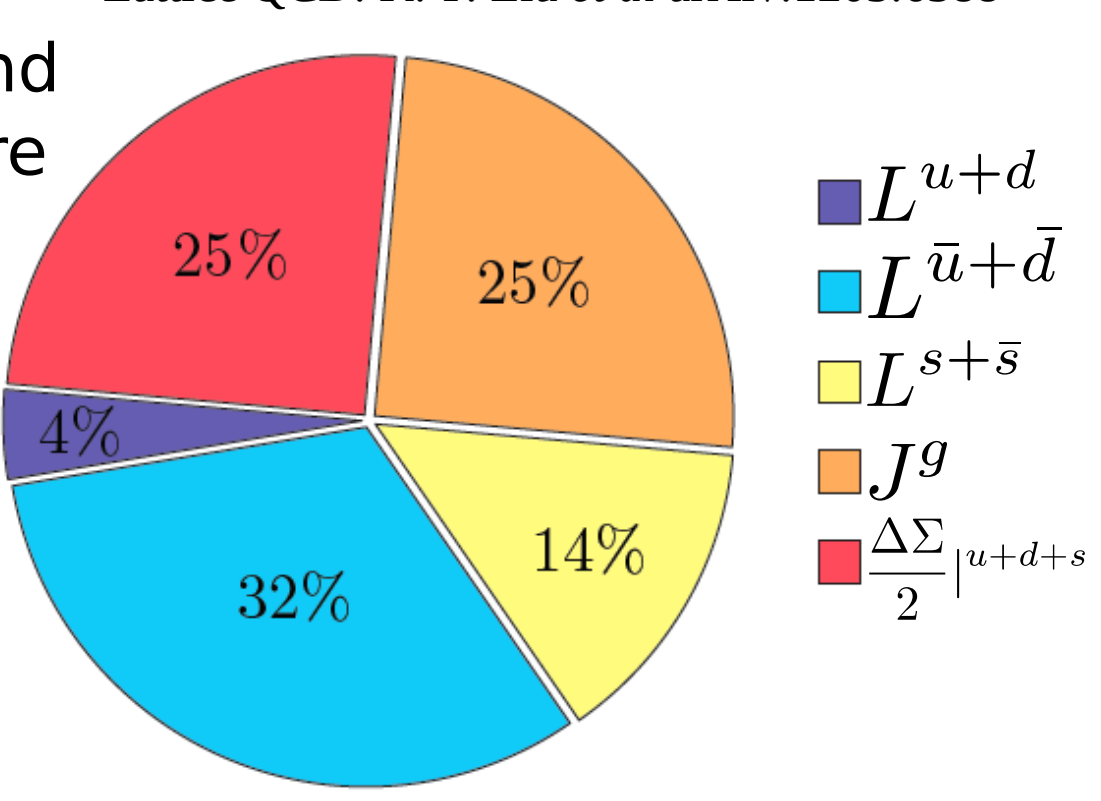

important to understand theoretically and experimentally

SpinQuest/E1039 will explore properties of the nucleon's spin composition by investigating correlation of the light antiquarks motion relative to the nucleon spin 


\section{HINTS OF NON-ZERO OAM}

sea quarks flavor asymmetry observation E866/NuSea: enhanced $\bar{d} / \bar{u}$

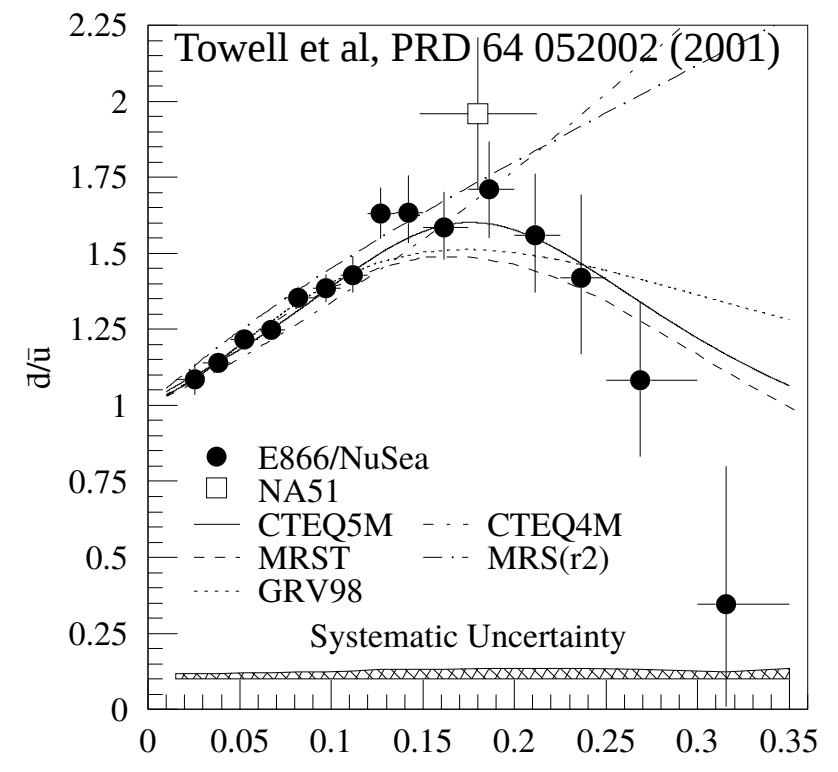

pion cloud model as possible interpretation $|p\rangle \propto\left|p_{0}\right\rangle+\left|n \pi^{+}\right\rangle+\left|\Delta^{++} \pi^{-}\right\rangle+\ldots$
E906/SeaQuest: $\sigma_{p d} / 2 \sigma_{p p}$

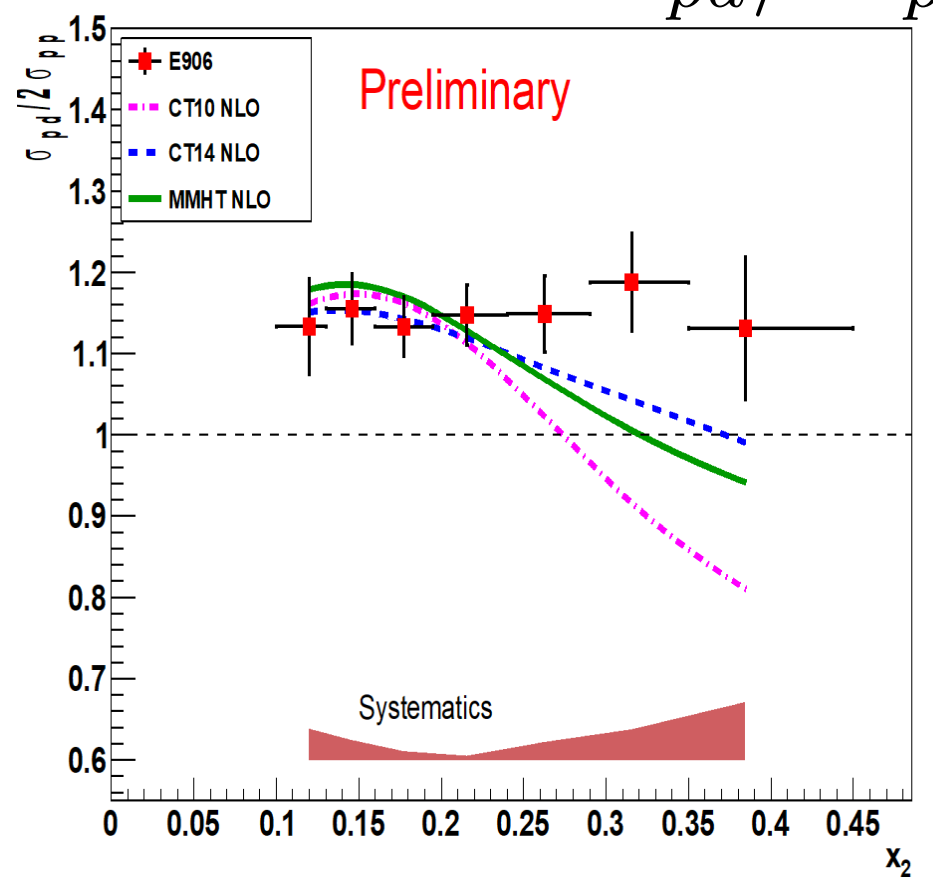

needs to conserve parity: pions negative parity requires $L=1$

if correct description - $|u \bar{d}\rangle$ possess OAM

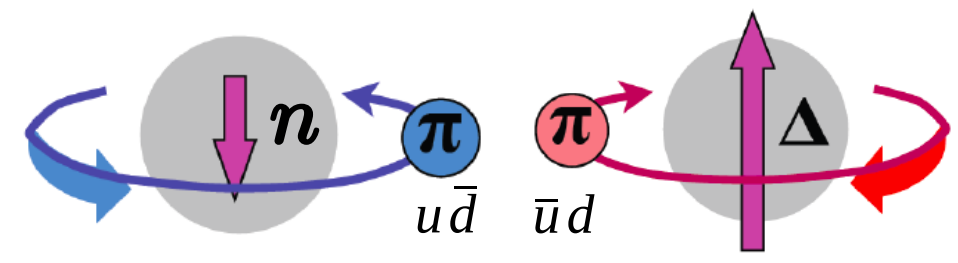

E866 results might point to sea quarks OAM 


\section{SIVERS FUNCTION}

explore correlation of quark motion relative to nucleon spin direction

eight leading order Transverse Momentum Dependent distribution functions (TMDs)

Sivers function - transverse momentum distribution of unpolarized quarks in a transversely polarized proton

$$
f_{1 T}^{\perp}\left(x, k_{T}\right)=\bigodot-\bigodot
$$

proposed to explain results of experiments such as SSA in $p p^{\uparrow} \rightarrow \pi X$ (E704)

- points to possible intrinsic $k_{T}$ imbalance; leads to asymmetry

if sea-quark Sivers asymmetry is non-zero, then sea quarks have non-zero OAM

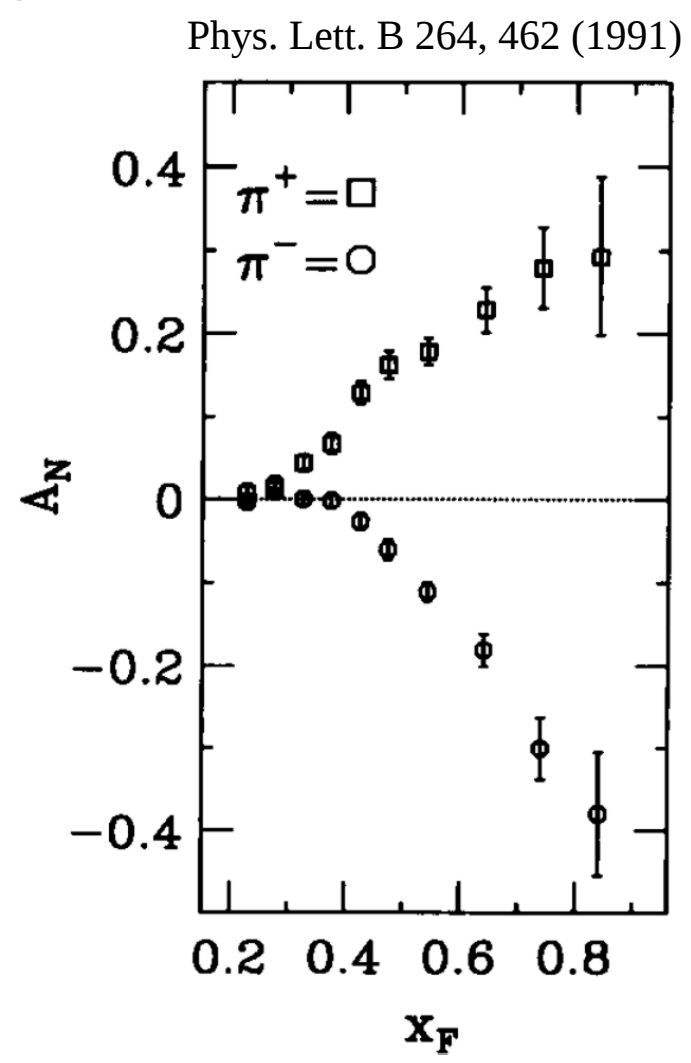




\section{ACCESSING SEA QUARK SIVERS FUNCTION}

$$
e+p^{\uparrow} \rightarrow e^{\prime} \pi X
$$

(1) Polarized Semi-Inclusive DIS

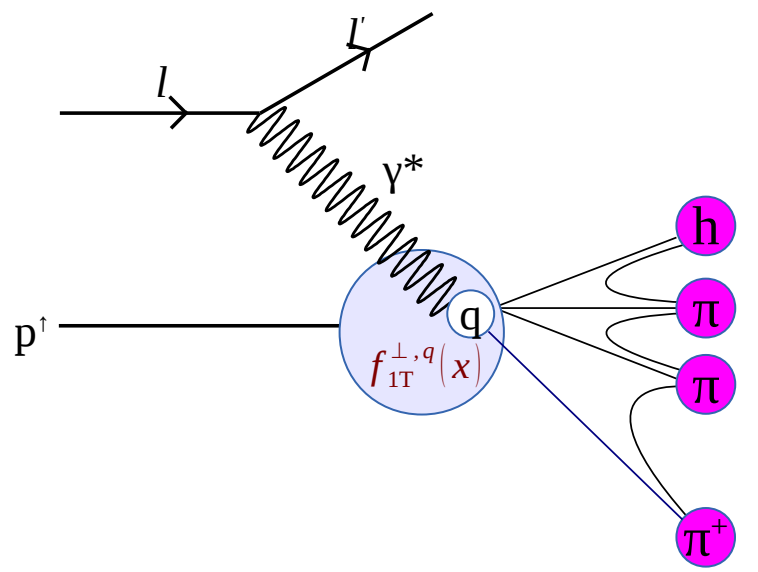

$A_{U T}^{S I D I S} \propto \frac{\sum_{q} e_{q}^{2} f_{1 T}^{\perp, q}(x) \otimes D_{1}^{q}(Z)}{\sum_{q} e_{q}^{2} f_{1}^{q}(x) \otimes D_{1}^{q}(Z)}$

- L-R asymmetry in hadron production

- quark to hadron fragmentation function

- valence-sea quark: mixed

$$
p+p^{\uparrow} \rightarrow \mu^{+} \mu^{-} X
$$

(2) Polarized Drell-Yan

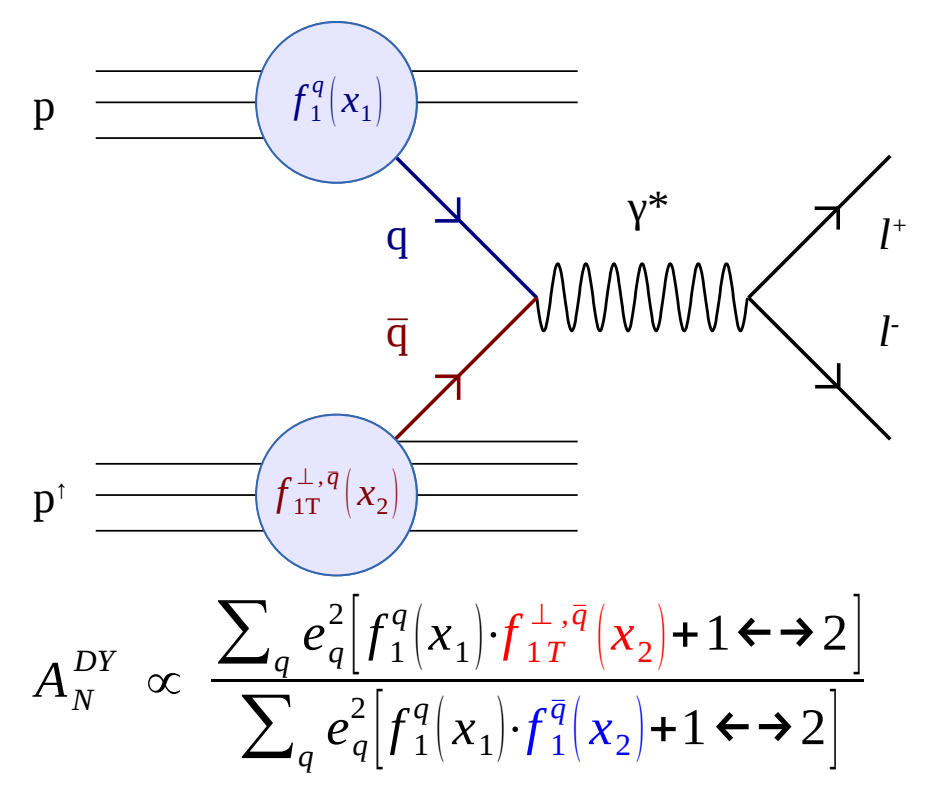

- L-R asymmetry in Drell-Yan production

- no fragmentation function

- valence-sea quark: isolated 


\section{ACCESSING SEA QUARK SIVERS FUNCTION}

$$
e+p^{\uparrow} \rightarrow e^{\prime} \pi X
$$

(1) Polarized Semi-Inclusive DIS

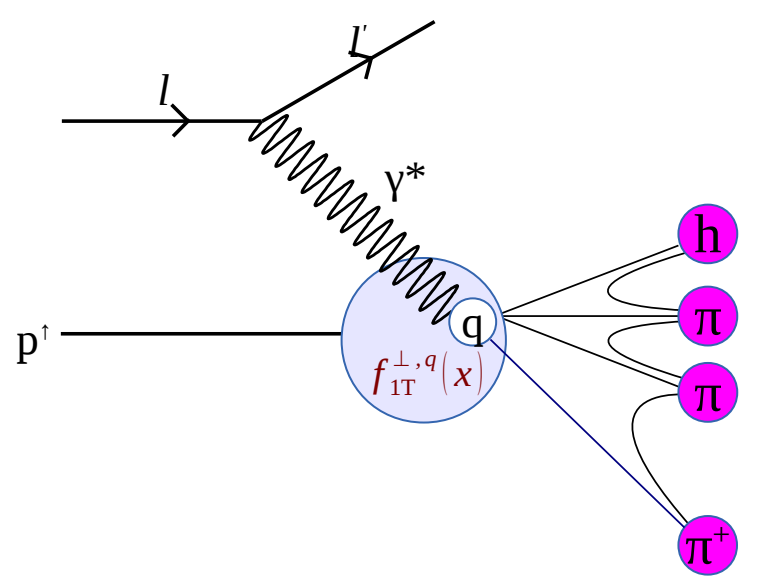

$A_{U T}^{\text {SIDIS }} \propto \frac{\sum_{q} e_{q}^{2} f_{1 T}^{\perp, q}(x) \otimes D_{1}^{q}(z)}{\sum_{q} e_{q}^{2} f_{1}^{q}(x) \otimes D_{1}^{q}(z)}$

- L-R asymmetry in hadron production

- quark to hadron fragmentation function

- valence-sea quark: mixed

$$
p+p^{\uparrow} \rightarrow \mu^{+} \mu^{-} X
$$

(2) Polarized Drell-Yan

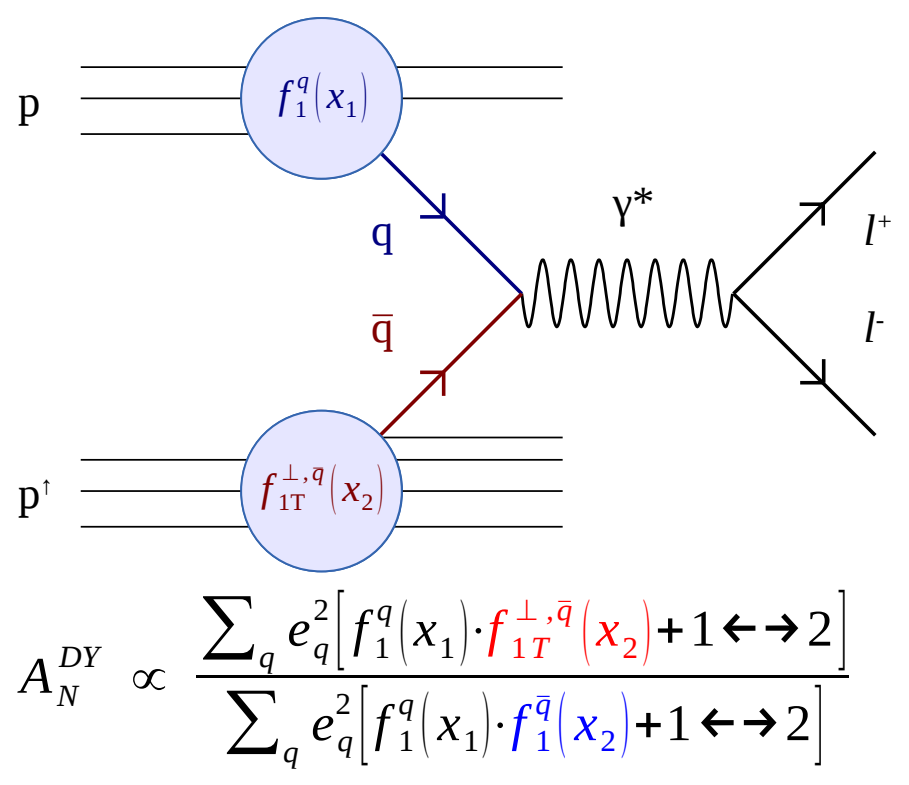

- L-R asymmetry in Drell-Yan production

- no fragmentation function

- valence-sea quark: isolated 


\section{ACCESSING SEA QUARK SIVERS FUNCTION}

has not been tried yet

- the only high statistics experiment sensitive to sea quarks at large $x$

\begin{tabular}{lcc}
\hline \hline quark & SIDIS & Drell-Yan \\
\hline valence & known & COMPASS \\
\hline sea & $\begin{array}{c}\text { poor } \\
\text { sensitivity }\end{array}$ & $\begin{array}{c}\text { unknown } \\
\text { E1039 }\end{array}$ \\
\hline \hline
\end{tabular}

selects sea quark from target

$\frac{d^{2} \sigma}{d x_{b} d x_{t}}=\frac{4 \pi \alpha^{2}}{9 x_{b} x_{t}} \frac{1}{s} \sum_{i} e_{i}^{2}$

$$
\times\left\{q_{i}\left(x_{b}\right) \bar{q}_{i}\left(x_{t}\right)+\bar{q}_{i}\left(x_{b}\right) q_{i}\left(x_{t}\right)\right\}
$$

- for E1039 kinematic configuration first term dominates

measure Sivers asymmetry for both

- $\bar{u}(x), \bar{d}(x)$

- determine possible flavor asymmetry

$$
p+p^{\uparrow} \rightarrow \mu^{+} \mu^{-} X
$$

(2) Polarized Drell-Yan

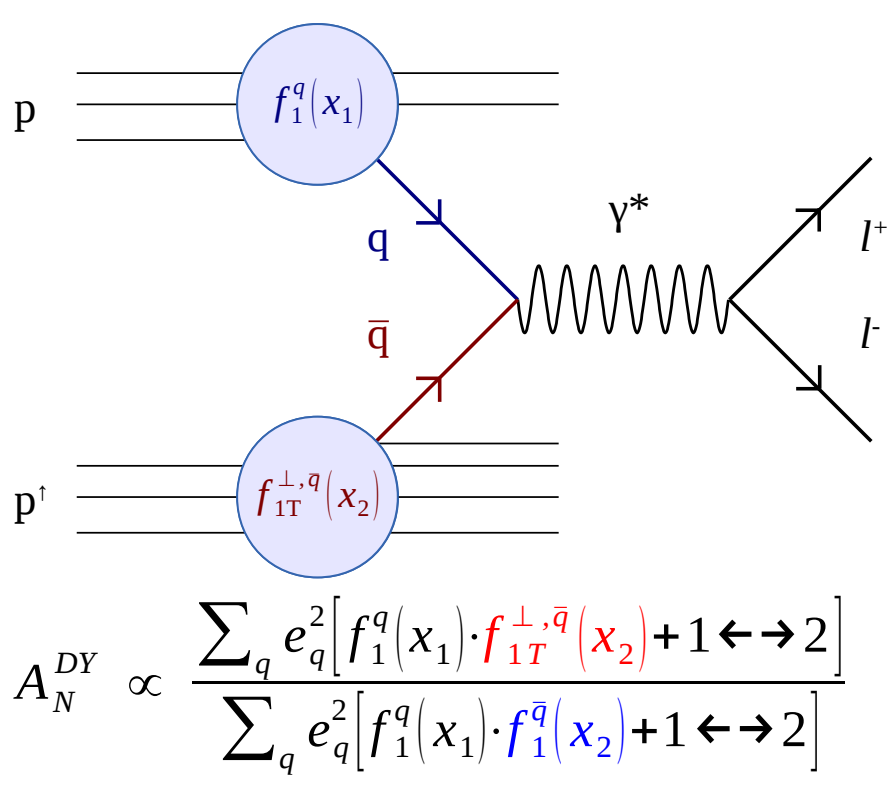

- L-R asymmetry in Drell-Yan production

- no fragmentation function

- valence-sea quark: isolated 


\section{FERMILAB E1039 EXPERIMENT}

asymmetry estimates (E1039 proposal)

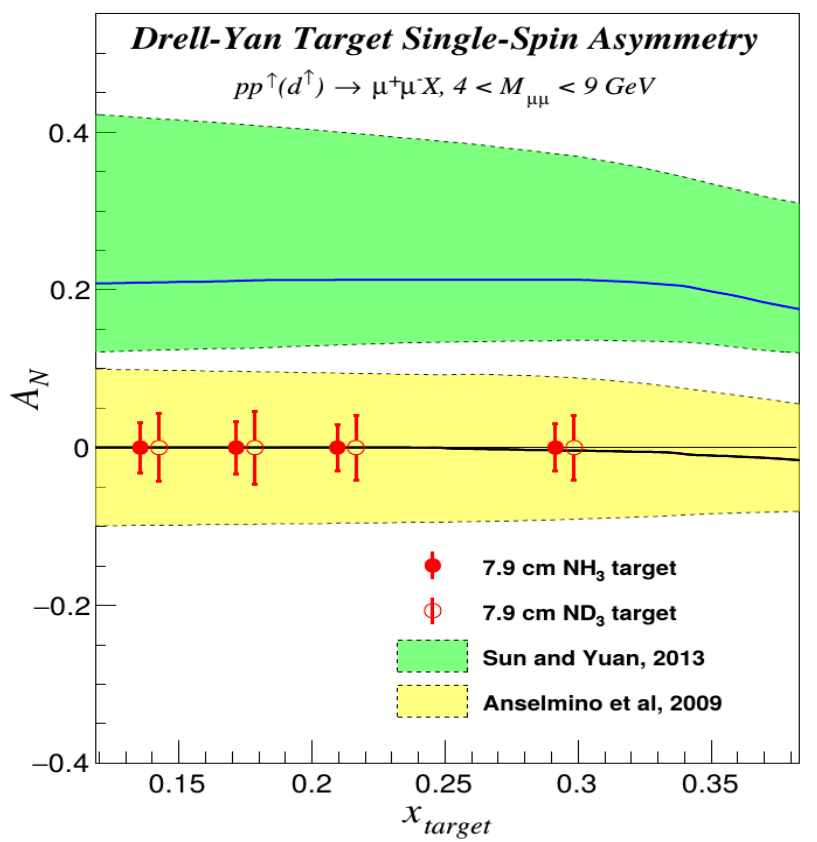

more recent calculations
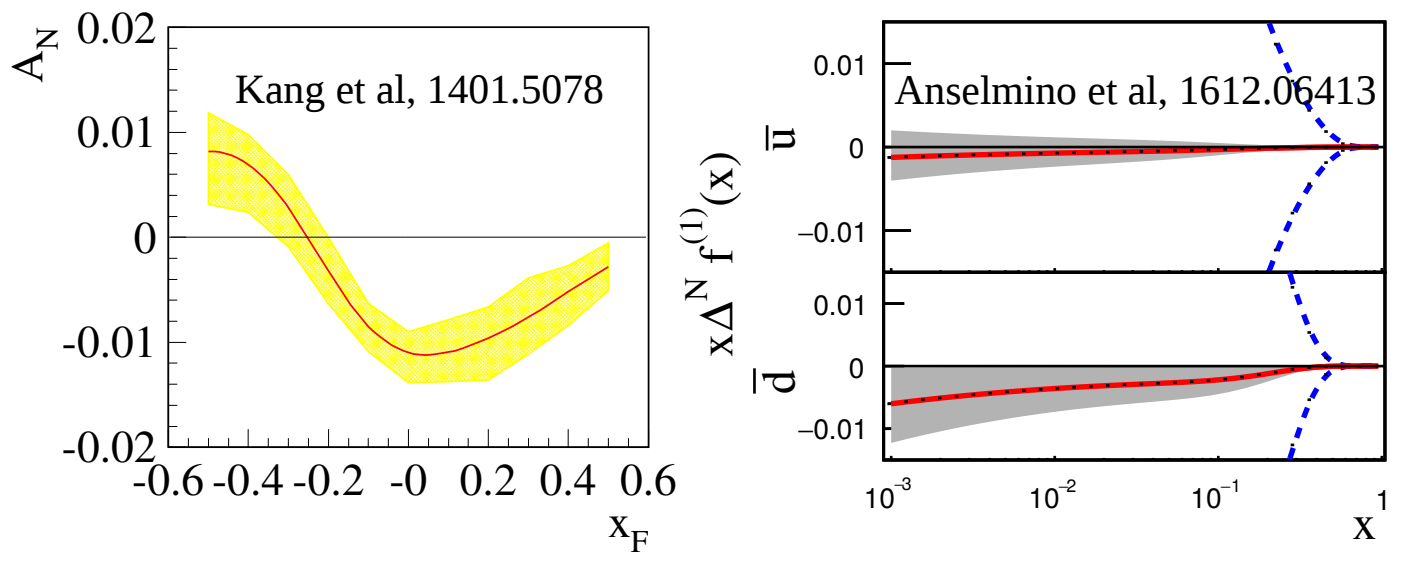

- sign and value of sea quark Sivers asymmetry

- sea quark Sivers flavor dependence

- if non-zero, "smoking gun" evidence for sea quark OAM

\section{statistics estimates and kinematics coverage}

\section{$\begin{array}{lllll}\text { Range } x_{2} & \text { Mean } x_{2} & N \text { events } p & \Delta A & p\end{array} \quad \mathrm{~N}$ events $n \quad \Delta A \% n$}

$\begin{array}{llllll}0.1-0.16 & .139 & 5.0 \times 10^{4} & 3.2 & 5.8 \times 10^{4} & 5.4 \\ 0.16-0.19 & 0.175 & 4.5 \times 10^{4} & 3.3 & 5.2 \times 10^{4} & 5.7 \\ 0.19-0.24 & 0.213 & 5.7 \times 10^{4} & 2.0 & 6.6 \times 10^{4} & 5.0 \\ 0.24-0.6 & 0.295 & 5.5 \times 10^{4} & 3.0 & 6.4 \times 10^{4} & 5.1\end{array}$




\section{FERMILAB E1039 EXPERIMENT}

O FNAL $120 \mathrm{GeV}$ proton beam

- $\sqrt{S}=15.5 \mathrm{GeV}$

- $5 \times 10^{12} \mathrm{p} / \mathrm{spill} ; 4.3 \mathrm{~s} / \mathrm{min}$

- $7.7 \times 10^{17}$ protons on target/year
O SeaQuest E906 spectrometer

- 4 tracking station, trigger hodoscope

- focusing \& analyzing magnets

- iron dump

O LANL/UVA polarized target

- solid $\mathrm{NH}_{3}, \mathrm{ND}_{3}$

- $5 \mathrm{~T}$ field, $1 \mathrm{~K}$ fridge

- $\mu$-wave freq. P flip

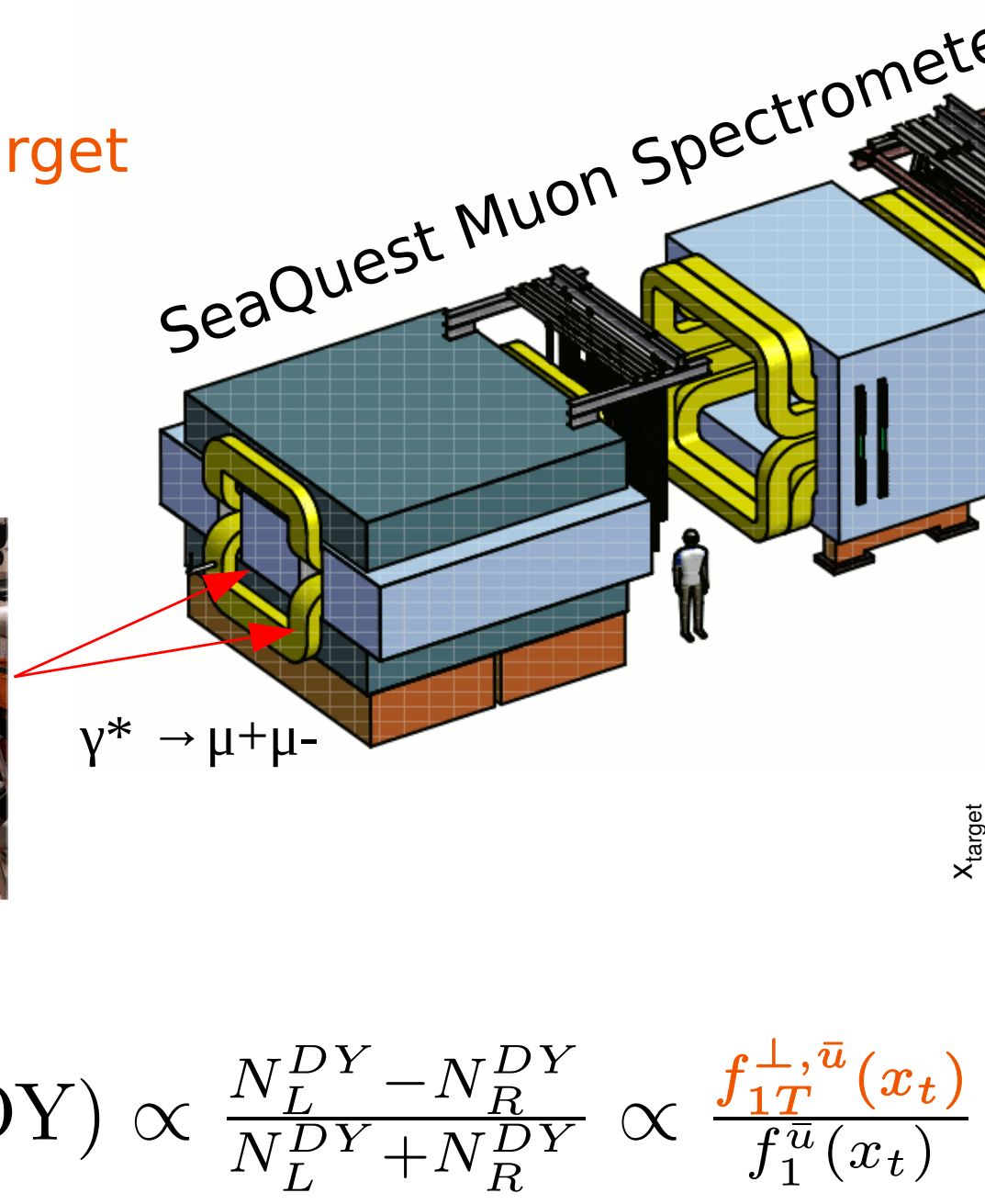

$A_{N}\left(p_{\text {beam }}+p_{\text {trg }}^{\uparrow}\right.$ $\rightarrow \mathrm{DY}) \propto \frac{N_{L}^{D Y}-N_{R}^{D Y}}{N_{L}^{D Y}+N_{R}^{D Y}}$ $\frac{\text { ENAL proton beam }}{E_{\text {beam }}}=120 \mathrm{GeV}$

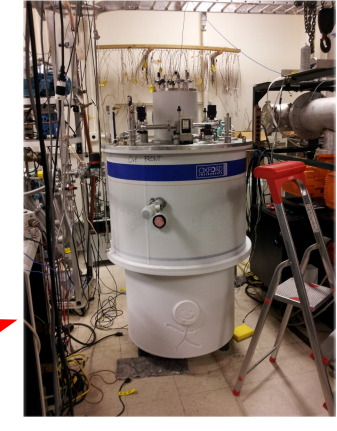

M. Yurov, LANL

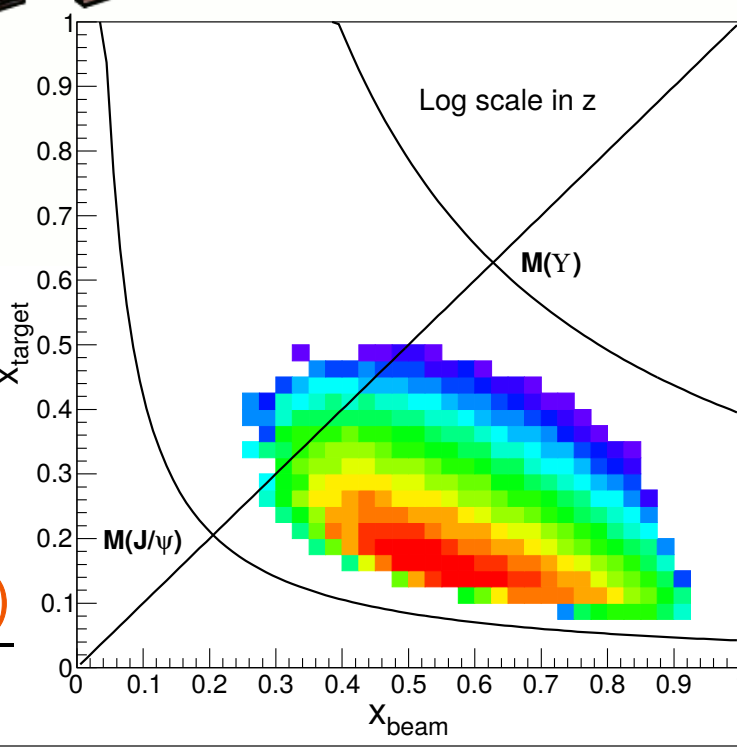

$9 / 17$ 


\section{STATUS and MILESTONES}

The experiment is fully funded by DOE, Stage-2 approval: 05/2018. 


\section{STATUS and MILESTONES}

major modifications in experimental hall

- beamline: new collimator

- new radiation shielding design

- new cryo platform for polarized target infrastructure

- polarized target cave: new location $300 \mathrm{~cm}$ upstream of FMAG

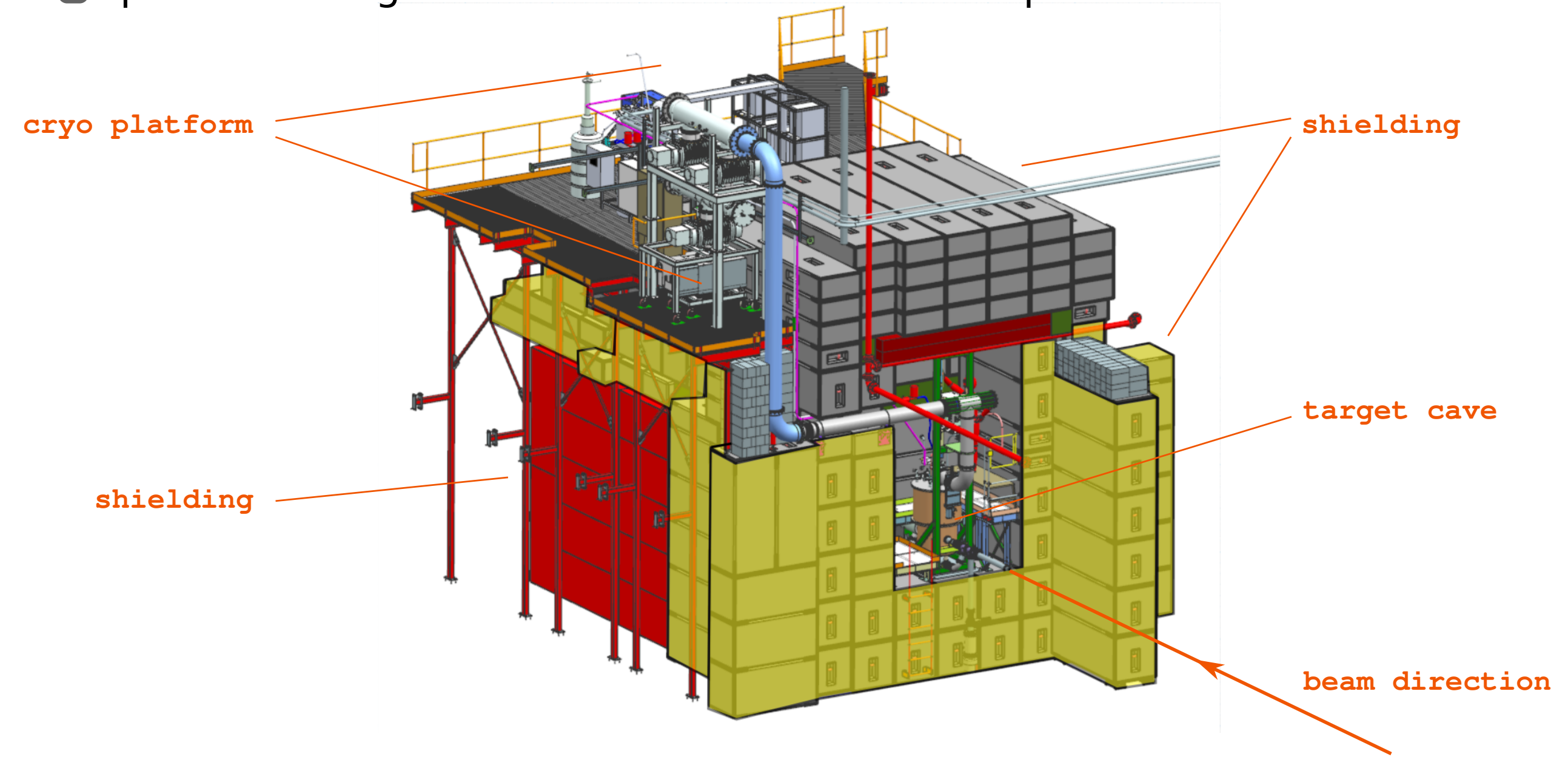




\section{STATUS and MILESTONES}

special thanks to Fermilab support

- beamline: new collimator

- new radiation shielding design

- new cryo platform for polarized target infrastructure

- polarized target cave: new location $300 \mathrm{~cm}$ upstream of FMAG

NM3: looking downstream

NM4: looking upstream

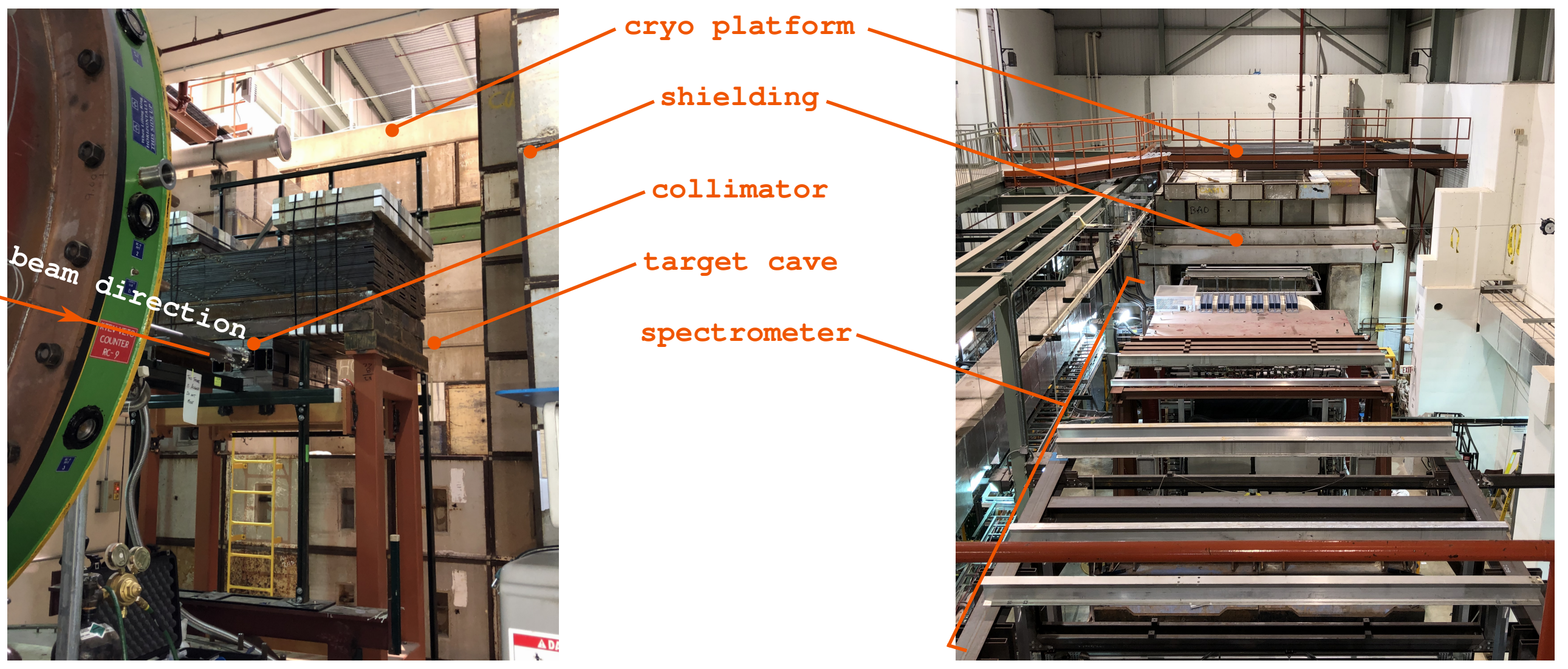

M. Yurov, LANL 


\section{STATUS and MILESTONES}

new polarized target construction: LANL and UVA effort

- rebuild magnet; change field direction

- modify $1 \mathrm{~K}$ refrigerator and insert

- new pump set; high cooling capacity

- new high power microwave source

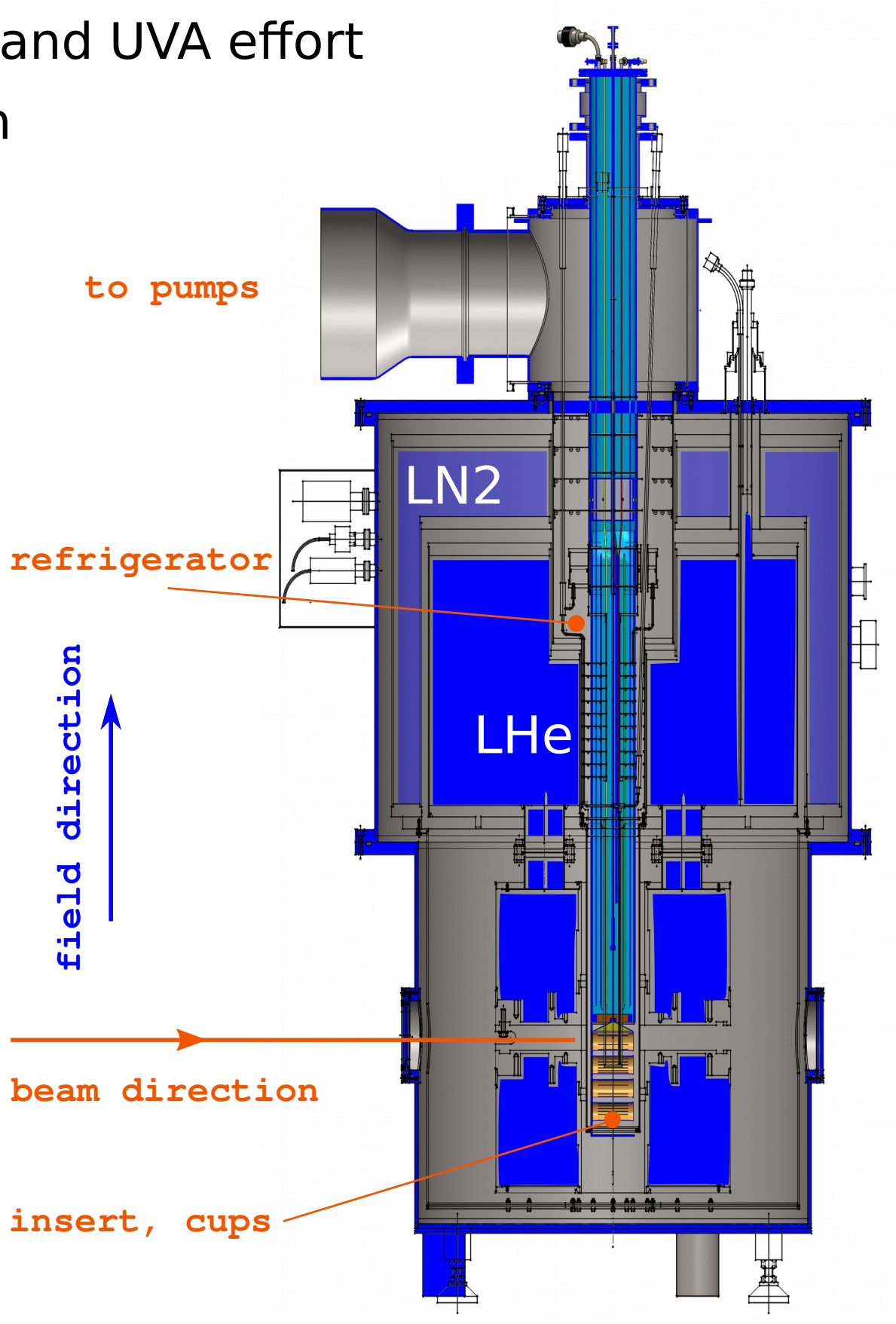




\section{STATUS and MILESTONES}

new polarized target construction: LANL and UVA effort

- rebuild magnet; change field direction

- modify $1 \mathrm{~K}$ refrigerator and insert

- new pump set; high cooling capacity

- new high power microwave source

new NMR system

- replacing Liverpool Q-meter

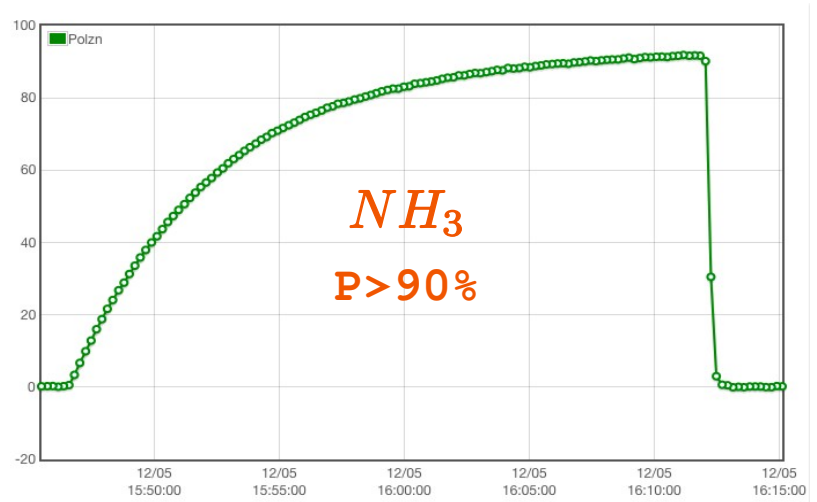

- double wide VME module

- electronic LCR and phase tune

- cold NMR

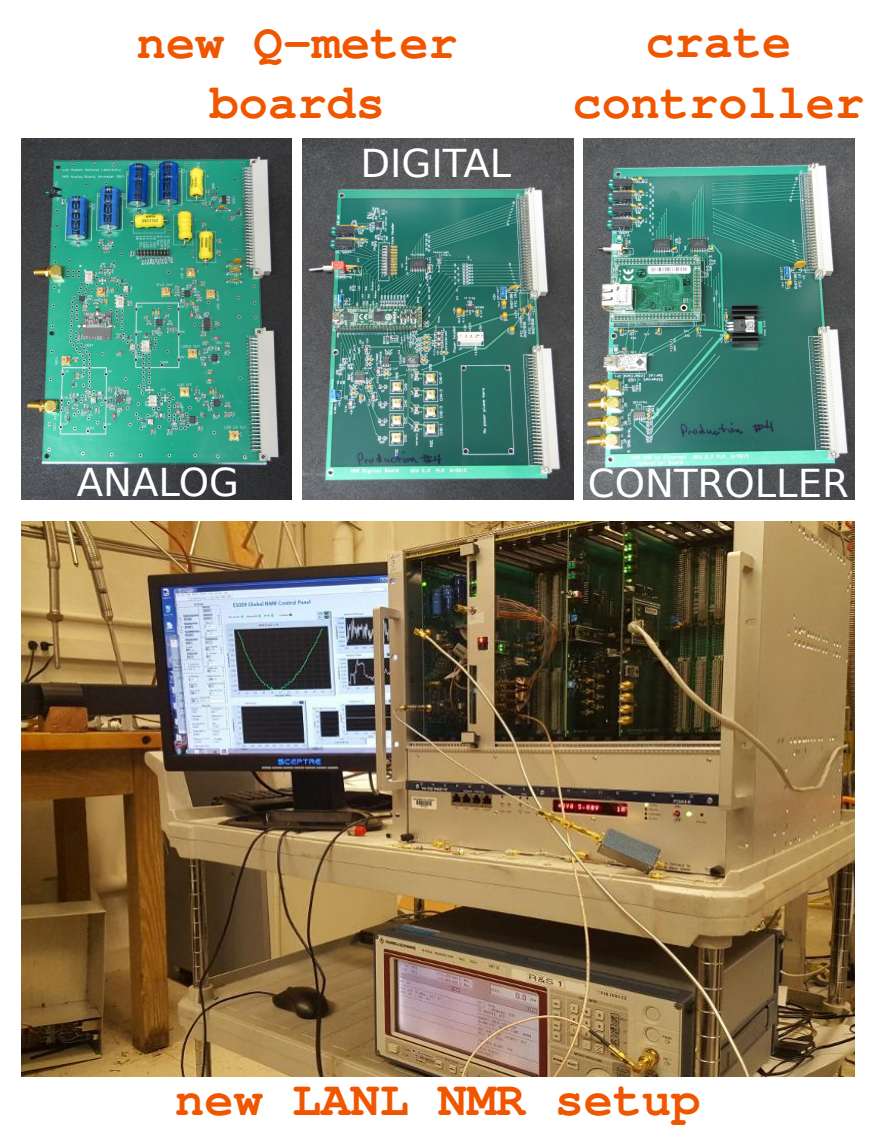




\section{STATUS and MILESTONES}

new polarized target construction: LANL and UVA effort

- rebuild magnet; change field direction

- modify $1 \mathrm{~K}$ refrigerator and insert

- new pump set; high cooling capacity

- new high power microwave source

new NMR system

- replacing Liverpool Q-meter

- double wide VME module

- electronic LCR and phase tune

- cold NMR

He liquefier for liquid helium recirculation

- constructed by "Quantum technologies"

- 200L/day capacity

inherit spectrometer from SeaQuest E906 experiment

- dedicated vertex trigger

- collaboration wide effor to prepare detectors/DAQ for experiment 


\section{STATUS and MILESTONES}

Current timeline:

May, 2018

Nov, 2018

June, 2019

"Today"

June/July, 2019

Summer, 2019

Fall, 2019

2019-2021

E906 decommissioning

last UVA target cooldown

target is shipped to FNAL

installation is in progress 


\section{SpinQuest/E1039 COLLABORATION}

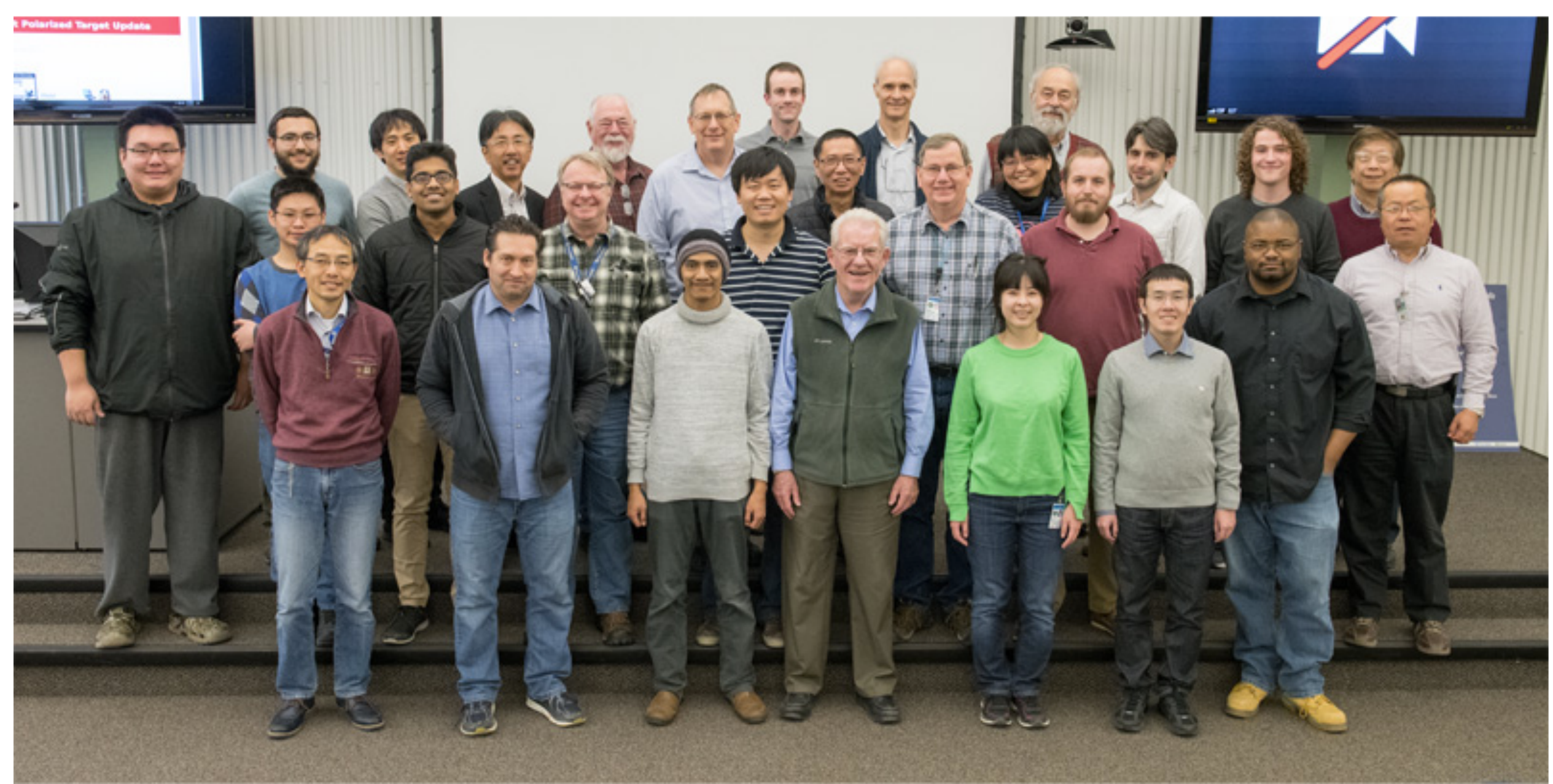

Contact Spokespersons:

Kun Liu (liuk@fnal.gov) - LANL Dustin Keller (dustin@jlab.org) - UVA

Learn more about SpinQuest/E1039: https://spinquest.fnal.gov/

M. Yurov, LANL 
THANK YOU

M. Yurov, LANL 